\title{
Probabilistic Distance, Capacity Clustering Location Model of a Semi-Obnoxious Facility, a Real Case of Tafo, Kumasi, Ghana
}

\author{
Dominic 0too1, Charles Sebil'2, Justice Amenyo Kessie'1, Ernest Larbi ${ }^{3}$ \\ ${ }^{1}$ Department of Mathematics \& Statistics, University of Energy \& Natural Resources, Sunyani, Ghana \\ ${ }^{2}$ Department of Mathematics \& Statistics, Kwame Nkrumah University of Science \& Technology, Kumasi, Ghana \\ ${ }^{3}$ Department of Mathematics \& Statistics, Catholic University College of Ghana, Sunyani, Ghana \\ Email: *dominic.otoo@uenr.edu.gh
}

How to cite this paper: Otoo, D., Sebil, C., Kessie, J.A. and Larbi, E. (2019) Probabilistic Distance, Capacity Clustering Location Model of a Semi-Obnoxious Facility, a Real Case of Tafo, Kumasi, Ghana. American Journal of Operations Research, 9, 146-160. https://doi.org/10.4236/ajor.2019.93009

Received: March 20, 2019

Accepted: May 28, 2019

Published: May 31, 2019

Copyright $\odot 2019$ by author(s) and Scientific Research Publishing Inc. This work is licensed under the Creative Commons Attribution International License (CC BY 4.0).

http://creativecommons.org/licenses/by/4.0/

\begin{abstract}
The collection of solid waste from third class communities in most developing countries is by skip containers, however, the location of these facilities has been done arbitrary without any mathematical considerations as to the number of customers the facility is serving, the distance one has to travel to access it and thereby making some of these residences to dump their refuse in gutters, streams and even burn them. In this paper we proposed an improved probabilistic distance, capacity clustering location model which takes into consideration the weight of solid waste from a customer and the capacity of the skip container to locate the skip container to serve a required number of customers based on the capacity constraint of the container. The model was applied on a real world situation and compared with the existing practice in terms of average distance customers had to travel to access the facility. Our results gave a well shorter average travel distance by customers, gave a number of skip containers needed in an area based on their waste generation per capita.
\end{abstract}

\section{Keywords}

Probabilistic, Facility Location, Capacity, Solid Waste, Skip Container, Clustering

\section{Introduction}

A Facility Location Problem (FLP) is "defined as in the positioning of a set of points (facilities in our case) within a given location space on the basis of the distribution of demand points (users) to be allocated to the facilities" [1]. Practically applied either in private or in public sector, these problems deal with 
strategic and long-term decisions involving huge investment costs. In general, once a facility is positioned, it produces effect, positive or negative, on the users (actual or potential), whose intensity is often thought-about depending on the mutual distance. Of course if the effects are positive (desirable facilities), effective positions for the facilities are expected as close as possible to the demand points. It is the case of public utility sites such as schools, hospitals, shops, banks, metro stations and so on. On the contrary, in case of negative effects (undesirable or obnoxious facilities) users wish that facilities are as far as possible. Examples of this kind concern power or nuclear plants, rubbish dumps. However, also in these cases, when facilities are too far from the demand points, additional costs have to be paid in terms of logistic costs. For this reason, it is necessary to find compromised solutions able to balance the different aspects [1]. Obnoxious facilities are facilities in which users no longer consider the facility desirable and try to have it close as possible but avoid the facility and stay away from it. In this paper, we would be looking at waste bins and their locations. Location of a semi-obnoxious facility such as solid waste containers (skip containers) has received scant attention especially in developing countries where customers would have to carry their waste to dump into these containers. Arbitrary location of such facilities in the communities has come with its own problems to society, some of which are the long distances, one has to cover to access the facility forcing people to dispose of solid waste into drains in their neighborhood, containers getting full and overflow in no time and thereby compelling people to throw their waste around the bin thereby creating unhealthy feeding grounds for domestic animals and possibly an outbreak of a disease (like reported here [2]) and unfriendly nature of the skip containers making it too difficult for children to dump solid waste into the bin. Each one of these problems increases the volume of filth in our communities, towns and cities rather than decreasing filth. It is rather therefore only reasonable that waste bins and skip containers can no longer be sited in arbitrary sites but must have behind them a concrete suggestion from a cluster model whose formulations and solution algorithms address the issue varying widely in terms of fundamental assumptions, mathematical complexity and computational performance [3]. In this paper we proposed an improved probabilistic distance, capacity clustering location model which takes into consideration the weight of solid waste from a customer and the capacity of the skip container to locate the skip container to serve a required number of customers based on the capacity constraint of the container taking Kumasi city as the case study. The results gave a shorter distance average travel distance for users or customers and also proposed the number of skip containers needed in the various zones considering the amount of waste generated in these zones.

\section{Study Area}

The research was conducted in Tafo, one of the nine sub-metropolitan assemblies in Kumasi, the second populated city in Ghana, West Africa. The nine sub-metropolitan areas are of Kumasi are: Suame, Manhyia, Bantama, Kwadaso, 
Nkyiaeso, Asokwa, Oforikrom, Subin and Tafo. The study area is the smallest of the nine sub metropolitan areas in terms of land area but it is the second highest generator of solid waste after Subin. The study area has a population of 157,226 with eleven communities within its domain. Four of these communities are categorized under class two (areas where we have fairly good road network but difficult for compactor trucks to collect waste) whiles the remaining seven are under class three (areas where we have bad road network and therefore impossible for proper collection of waste by heavy vehicles). The study area shares boundary with Manhyia to the east, Suame to the west and Subin to the south. The area generates about eighty-eight tones of solid waste a day. Our study considered five of the seven third class zones namely Old Tafo, Pankrono Dome, Pankrono West, Tafo Adompom and Ahenbronum constituting about 52\% of the area population. The area has the second largest market in the Kumasi metropolis, has a number of government assisted schools from basic to secondary levels and one government hospital. Figure 1 shows the map of Kumasi Metropolis where the study has been done.

\section{Literature Review}

Location of facilities be it semi or obnoxious has had its fair share in terms of research in literature, that notwithstanding there are still many areas that needs attention to improve the difficulties service customers go through to access a facility. This section takes a look into various research works that has gone into

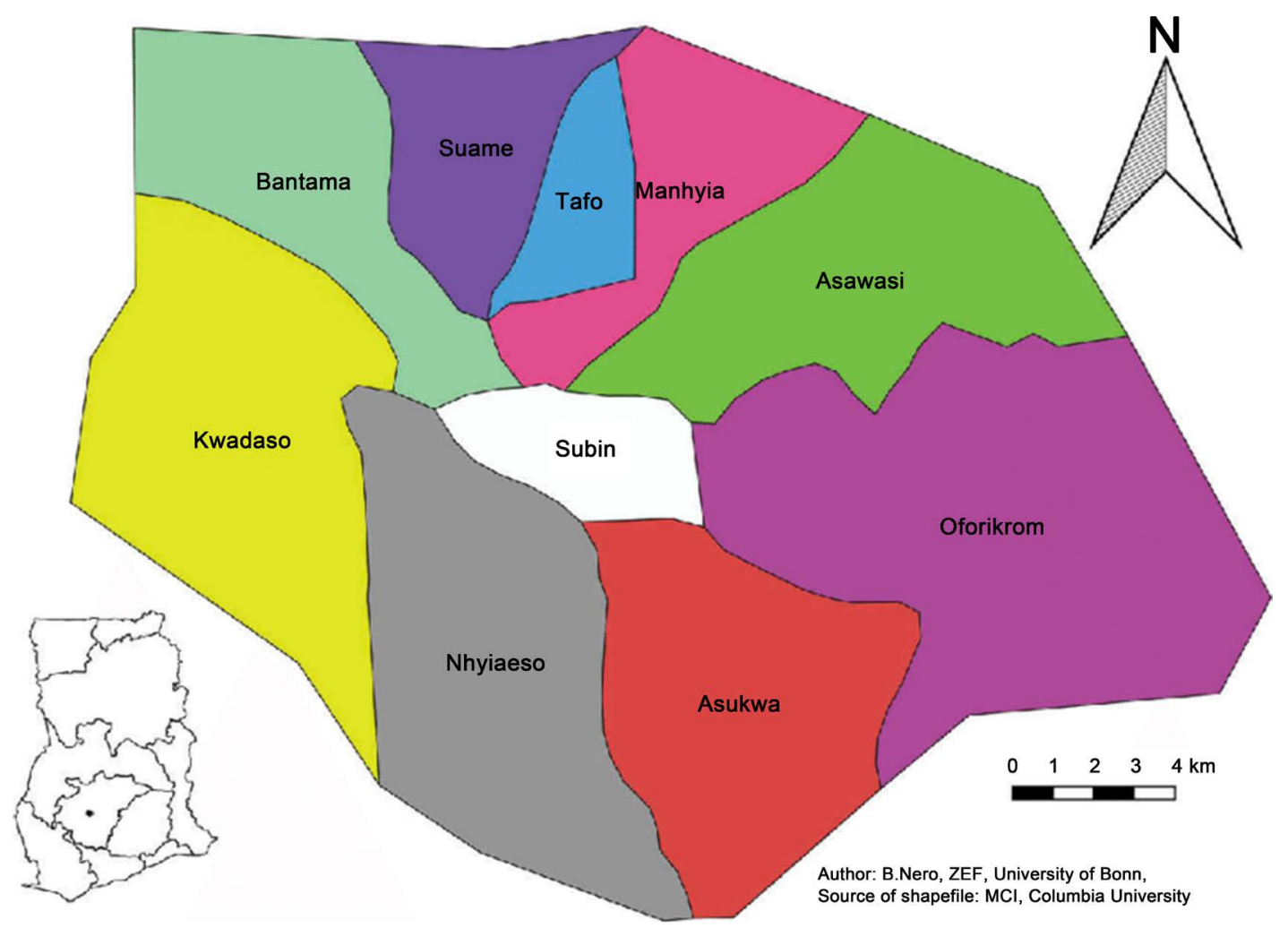

Figure 1. Map of Kumasi Metropolis. 
facility location of obnoxious and semi-obnoxious alike. A semi-obnoxious facility is a facility which is useful to the end users but has some level of unwelcome effect that produces environmental concerns to the same end user. [4] defined an obnoxious facility as one that generates a disservice to the people nearby while producing an intended product or service. [5] first introduced the rectilinear maximin problem for locating an obnoxious facility. They developed a solution procedure by dividing the feasible region into rectilinear sub-regions and solved using a linear programming problem for each of these sub regions. [6] presented a multi-objective mixed integer linear model for undesirable facility location. The objectives considered are cost minimization and equity maximization. [7] [8] presented a multi-objective mixed integer programming model for the location of hazardous material facilities including the choice of variable technology with cost, risk and equity as the main objective functions. [9] proposed a dynamic multi-period multi-objective capacitated mixed integer programming model for the location of sanitary landfills. [10] studied the location of semi obnoxious facilities as a discrete location problem on a network. Several bi-criteria models are presented considering two conflicting objectives, the minimization of the obnoxious effect and the maximization of the accessibility of the communities to the closest open facility. These objectives tried to optimize the average value over the entire communities' worst value is considered in two different ways, trying to optimize its average value over all the communities or trying to optimize its worst value. They used Euclidean distance to evaluate the obnoxious effect and the shortest distance path to evaluate the accessibility.

[11] presented a model for the location of semi obnoxious facilities and simultaneously routing the undesirable materials between facilities and communities alike. [12] developed two bi-criteria models for single allocation hub location problems. In both models they used total time as the first criteria to be minimized and used time minimization to process flow entering the hubs. They considered, total time as the second criteria in the first model and, in the second model, the maximum service time for the hubs is minimized. [13] studied a simplified model where clients will be assigned to (unreliable) facilities and reliable backup facilities if needed. [14] presented both scenario-based stochastic programming and a nonlinear mixed integer programming model and show that they are generally equivalent. Also, a constant-ratio approximation algorithm for the case where all failure rates are identical is proposed. [15] considered problems with a fortification budget in the first stage so that unreliable facilities can be fortified by hardening operations. Recently, this line of research is extended to investigate more general reliable network design problems. [16] considered a reliable multiple-echelon logistics network design problem where disruptions can happen in multiple echelons. [17] studied reliable hub-and-spoke network design problems in which hubs could be disrupted and affected flows will be rerouted through survived operational hubs. In their paper, [18] considered a multi-facility location problem in the presence of a line barrier with the starting 
point of the barrier uniformly distributed. Their objective was to locate $n$ new facilities among $m$ already existing facilities minimizing the summation of the weighted expected rectilinear barrier distances of the locations of new facilities and new and existing facilities. In their scholarly presentation, he sought to solve the problem of location where there are restrictions so that the restrictions prohibit establishment of new facilities in some specified regions. They designed a mixed-integer nonlinear programming model, which was conveniently transformed into a mixed-integer quadratic programming model. They also proposed two meta-heuristic algorithms, namely the genetic algorithm and the imperialist competitive algorithm for optimization for large problems. In their paper [9], an integer programming model was proposed to help decision makers in choosing the sites to locate the unsorted waste collection bins in a residential town, as well as the capacities of the bins to be located at each collection site. Their model helped in assessing tactical decisions through constraints that force each collection area to be capacitated enough to fit the expected waste to be directed to that area, while taking into account Quality of Service constraints from the citizens' point of view. Additionally, they proposed an effective constructive heuristic approach whose aim is to provide a good solution quality in an extremely reduced computational time. Computational results on data related to the city of Nardò, in the south of Italy, show that both exact and heuristic approaches provide consistently better solutions than that currently implemented, resulting in a lower number of activated collection sites, and a lower number of bins to be used [9].

\subsection{Maximum Insertion Probabilistic Model}

A location problem is to locate a facility, or facilities, to serve optimally a given set of customers. The customers are given by their coordinates and demands. The coordinates are points in $R^{n}$ (usually $n=2$ ), and the demands are positive numbers $q_{\dot{r}}$ Assuming $N$ customers, the data of the problem is a set of points (coordinates) $X=\left\{X_{1}, X_{2}, \cdots, X_{N}\right\}$ in $R^{n}$ and a corresponding set of positive weights (demands) $\left\{q_{1}, q_{2}, \cdots, q_{N}\right\}$. Using the Euclidean distance $d(X, Y)=\|X-Y\|$ between two points $X, Y$ in $R^{n}$. If the customers are served by $K$ facilities for a given $K$, we denote $X_{K}$ to be the set of customers assigned to the $K^{\text {th }}$-facility. The weighted sum of distance travelled by these customers is $\sum_{X_{i} \in \chi_{K}} \frac{1}{q_{i}}\left\|X_{i}-c_{K}\right\|$ where $c_{K}$ is the location of the $K^{\text {th }}$-facility. The customers $X=\left\{X_{1}, X_{2}, \cdots, X_{N}\right\}$, their demands $\left\{q_{1}, q_{2}, \cdots, q_{N}\right\}$ is to be located to facilities with centres $\left\{c_{1}, c_{2}, \cdots, c_{K}\right\}$ so as to minimize the weighted sum of distances travelled by all the customers to access the bin. $\min _{c_{1}, \cdots, c_{K}} \min _{X_{1}, \cdots, X_{K}} \sum_{k=1}^{K} \sum_{X_{1} \in \chi_{K}} \frac{1}{q_{i}}\left\|X_{i}-c_{K}\right\|$ and each container has a capacity $Q$, such that the sum of demands assigned to the $K^{\text {th }}$-facility cannot exceed it, i.e.

$$
\sum_{X_{i} \in \chi_{K}} q_{i} \leq Q_{K}
$$

Let a data set $D \subset R^{p}$ be partitioned into $K$ clusters $\left\{c_{k}: k=1,2, \cdots, K\right\}$, 
$D=\bigcup_{k=1}^{K} C_{k}$ and let $C_{k}$ be the centre of the cluster $C_{k}$ with capacity $Q_{K}$.

With each data point $X \in D$ and a cluster centre $C_{k}$, we denote:

- a distance $d_{k}\left(X, c_{k}\right)$ by $d_{k}(X)$

- a probability of membership in $c_{k}$ by $P_{k}(X)$ for each $X \in D$ and each cluster $C_{k}$,

$$
\frac{P_{k}(X) d_{k}(X)}{Q_{k}}=\text { Constant }
$$

depending on $X$ and independent of the cluster $k$.

Given the cluster centers $\left\{C_{1}, C_{2}, \cdots, C_{k}\right\}$, let $X$ be a data point and let $\left\{d_{k}(X): k=1,2, \cdots, k\right\}$ be its distances from the given centers.

From Equation (3.2) using $i$ and $k P_{i}(X) \frac{d_{i}(X)}{Q_{i}}=P_{k}(X) \frac{d_{k}(X)}{Q_{k}}$

$$
\begin{gathered}
\text { Since } \sum_{i=1}^{K} P_{i}(X)=1, \quad P_{k}(X) \sum_{i=1}^{K}\left(\frac{\frac{d_{k}(X)}{Q_{k}}}{\frac{d_{i}(X)}{Q_{i}}}\right)=1 \\
P_{k}(X)=\frac{\prod_{j \neq k} \frac{d_{j}(X)}{Q_{j}}}{\sum_{i=1}^{K} \prod_{j \neq i} \frac{d_{j}(X)}{Q_{j}}}, k=1,2, \cdots, K
\end{gathered}
$$

For two clusters, i.e. $K=2$

$$
P_{1}=\frac{\frac{d_{2}(X)}{Q_{2}}}{\frac{d_{1}(X)}{Q_{1}}+\frac{d_{2}(X)}{Q_{2}}}, \quad P_{2}=\frac{\frac{d_{1}(X)}{Q_{1}}}{\frac{d_{1}(X)}{Q_{1}}+\frac{d_{2}(X)}{Q_{2}}}
$$

For three clusters i.e. $K=3$

$$
P_{1}=\frac{\frac{d_{2}(X) d_{3}(X)}{Q_{2} \times Q_{3}}}{\frac{d_{1}(X) d_{2}(X)}{Q_{1} \times Q_{2}}+\frac{d_{1}(X) d_{3}(X)}{Q_{1} \times Q_{3}}+\frac{d_{2}(X) d_{3}(X)}{Q_{2} \times Q_{3}}}
$$

Denoting the constant term in Equation (3.2) by $D(X)$, function in $X$, $\frac{P_{k}(X) d_{k}(X)}{Q_{k}}=D(X)$

$$
\begin{aligned}
& P_{k}(X)=\frac{D(X)}{\frac{d_{k}(X)}{Q_{k}}}, k=1,2,3, \cdots, K \\
& \text { Since } \sum_{i=1}^{K} P_{k}(X)=1
\end{aligned}
$$




$$
D(X)=\frac{\prod_{j=1}^{K} \frac{d_{j}(X)}{Q_{j}}}{\sum_{i=1}^{K} \prod_{j \neq i} \frac{d_{j}(X)}{Q_{j}}}
$$

For two clusters, i.e. $K=2$,

$$
D(X)=\frac{\frac{d_{1}(X) d_{2}(X)}{Q_{1} Q_{2}}}{\frac{d_{1}(X)}{Q_{1}}+\frac{d_{2}(X)}{Q_{2}}}
$$

For three clusters, i.e. $K=3$;

$$
D(X)=\frac{\frac{d_{1}(X) d_{2}(X) d_{3}(X)}{Q_{1} Q_{2} Q_{3}}}{\frac{d_{1}(X)}{Q_{1}}+\frac{d_{2}(X)}{Q_{2}}+\frac{d_{3}(X)}{Q_{3}}}
$$

Therefore for the entire data set $D$ is the sum of (3.6) over all points, and is a function of the $K$ cluster centers,

$$
F\left(c_{1}, c_{2}, \cdots, c_{k}\right)=\sum_{i=1}^{N} \frac{\prod_{k=1}^{K} d_{k}\left(X_{i}, c_{k}\right)}{\sum_{t=1}^{K} \prod_{j \neq t} d_{j}\left(X_{i}, c_{j}\right)}
$$

\subsection{An Extremal Principle}

Consider a case of two clusters, using $X$ to be the given data point with distances $d_{1}(X), d_{2}(X)$ to the cluster centers and a known cluster sizes $Q_{1}$ and $Q_{2}$ known. The probabilities in (3) are the optimal solution $P_{1}, P_{2}$ of the extremal problem.

$$
\begin{aligned}
\text { Minimize } & \left\{\frac{d_{1}(x) P_{1}^{2}}{Q_{1}}+\frac{d_{2}(x) P_{2}^{2}}{Q_{2}}\right\} \\
\text { Subject to } & P_{1}+P_{2}=1 \\
& P_{1}, P_{2} \geq 0
\end{aligned}
$$

The Lagrangian of the problem is:

$$
L\left(P_{1}, P_{2}, \lambda\right)=\frac{d_{1}(x) P_{1}^{2}}{Q_{1}}+\frac{d_{2}(x) P_{2}^{2}}{Q_{2}}-\lambda\left(P_{2}+P_{1}-1\right)
$$

Setting the partial derivatives with respect to $P_{1}, P_{2}$ to zero, we have $P_{1} d_{1}(x)=P_{2} d_{2}(x)$.

Substitute the probabilities in (3.4) in (3.10) we have

$L^{*}\left(P_{1}(x), P_{2}(x), \lambda\right)=\frac{\frac{d_{1}(x) d_{2}(x)}{Q_{1} Q_{2}}}{\frac{d_{1}(x)}{Q_{1}}+\frac{d_{2}(x)}{Q_{2}}}$ which is the same as the joint distance function obtained in Equation (3.9). 
The extremal problem for the entire data set $D=\left\{X_{1}, X_{2}, \cdots, X_{N}\right\} \subset R^{n}$ Minimize

$$
\sum_{i=1}^{N}\left(\frac{d_{1}\left(x_{i}\right) p_{1}\left(x_{i}\right)^{2}}{Q_{1}}+\frac{d_{2}\left(x_{i}\right) p_{2}\left(x_{i}\right)^{2}}{Q_{2}}\right)
$$

Subject to: $p_{1}\left(x_{i}\right)+p_{2}\left(x_{i}\right)=1$

$$
p_{1}\left(x_{i}\right), p_{2}\left(x_{i}\right) \geq 0, i=1,2,3, \cdots, N
$$

where $p_{1}\left(x_{i}\right), p_{2}\left(x_{i}\right)$ are the cluster probabilities at $x_{i}$ and $d_{1}\left(x_{i}\right), d_{2}\left(x_{i}\right)$ are the corresponding distances. The problem separates into $N$ as in (3.11) and its optimal value is $\sum_{i=1}^{N} \frac{\frac{d_{1}\left(x_{i}\right) d_{2}\left(x_{i}\right)}{Q_{1} Q_{2}}}{\frac{d_{1}\left(x_{i}\right)}{Q_{1}}+\frac{d_{2}\left(x_{i}\right)}{Q_{2}}}$ the sum of the joint distance function of all points.

\subsection{Cluster Centers}

Writing Equation (3.11) as a function of the cluster centers $C_{1}, C_{2}$

$$
f\left(C_{1}, C_{2}\right)=\sum_{i=1}^{N}\left(\frac{d_{1}\left(x_{i}, C_{1}\right) p_{1}\left(x_{i}\right)^{2}}{Q_{1}}+\frac{d_{2}\left(x_{i}, C_{2}\right) p_{2}\left(x_{i}\right)^{2}}{Q_{2}}\right) .
$$

For Euclidean distance $d_{k}\left(x, c_{k}\right)=\left\|x-c_{k}\right\|, k=1,2$ so that

$$
f\left(C_{1}, C_{2}\right)=\sum_{i=1}^{N}\left(\frac{\left\|x_{i}-C_{1}\right\| p_{1}\left(x_{i}\right)^{2}}{Q_{1}}+\frac{\left\|x_{i}-C_{2}\right\| p_{2}\left(x_{i}\right)^{2}}{Q_{2}}\right)
$$

and look for centers $C_{1}, C_{2}$ that minimizes $f$ and the probabilities $p_{1}\left(x_{i}\right), p_{2}\left(x_{i}\right)$ are given for $i=1,2,3, \cdots, N$ and with an assumption that the cluster centers $C_{1}, C_{2}$ do not coincide with any of the points $i=1,2,3, \cdots, N$

From the assumption above, the gradient of Equation (3.12) with respect to $c_{k}$ is

$$
\begin{aligned}
\nabla_{c} f\left(c_{1}, c_{2}\right) & =-\sum_{i=1}^{N} \frac{x_{i}-c_{k}}{\left\|x_{i}-c_{k}\right\|} \times p_{k}\left(x_{i}\right)^{2} \\
& =-\sum_{i=1}^{N} \frac{x_{i}-c_{k}}{d_{k}\left(x_{i}, c_{k}\right)} \times p_{k}\left(x_{i}\right)^{2}, k=1,2
\end{aligned}
$$

Setting the gradient to zero, and grouping like terms, we have

$$
\begin{gathered}
\sum_{i=1}^{N}\left(\frac{p_{k}\left(x_{i}\right)^{2}}{d_{k}\left(x_{i}, c_{k}\right)}\right) x_{i}=\left(\sum_{i=1}^{N} \frac{p_{k}\left(x_{i}\right)^{2}}{d_{k}\left(x_{i}, c_{k}\right)}\right) c_{k} \\
C_{k}=\sum_{i=1}^{N}\left(\frac{u_{k}\left(x_{i}\right)}{\sum_{j=1}^{N} u_{k}\left(x_{j}\right)}\right) x_{i}
\end{gathered}
$$

where $u_{k}=\frac{p_{k}\left(x_{i}\right)^{2}}{d_{k}\left(x_{i}, c_{k}\right)}$ for $k=1,2$ 
Giving the minimizers as

$$
C_{1}=\sum_{i=1}^{N}\left(\frac{u_{1}\left(x_{i}\right)}{\sum_{j=1}^{N} u_{1}\left(x_{j}\right)}\right) x_{i}, C_{2}=\sum_{i=1}^{N}\left(\frac{u_{2}\left(x_{i}\right)}{\sum_{j=1}^{N} u_{2}\left(x_{j}\right)}\right) x_{i}
$$

where

$$
u_{1}=\frac{p_{1}\left(x_{i}\right)^{2}}{d_{1}\left(x_{i}, c_{1}\right)}, u_{2}=\frac{p_{2}\left(x_{i}\right)^{2}}{d_{2}\left(x_{i}, c_{2}\right)}
$$

For a function of $K$ cluster centers

$f\left(C_{1}, C_{2}, \cdots, C_{K}\right)=\sum_{k=1}^{K} \sum_{i=1}^{N}\left(\frac{d_{k}\left(x_{i}, C_{k}\right) p_{k}\left(x_{i}\right)^{2}}{Q_{k}}\right)$ an analog of (3.13) then by the results of Equation (3.2) the minimizers of $f$ is

$$
C_{k}=\sum_{i=1}^{N}\left(\frac{u_{k}\left(x_{i}\right)}{\sum_{j=1}^{N} u_{k}\left(x_{j}\right)}\right) x_{i} \text { with } u_{k}=\frac{p_{k}\left(x_{i}\right)^{2}}{d_{k}\left(x_{i}, c_{k}\right)} \text { for } k=1,2, \cdots, K
$$

\section{Algorithm for Probabilistic Distance Location Model}

The major steps involved in the formation of the algorithm are described below.

1) Calculation of number of skip containers

The number of skip containers $(k)$ needed in each zone is dependent on demands $\left(q_{i}\right)$ of the customers and the capacity of the $(Q)$ of the container; $k=\frac{\sum_{i=1}^{N} q_{i}}{Q}$.

\section{2) Selection of initial cluster centers}

- The $x$-coordinates of all the customers are arranged in ascending order. The highest $x$-coordinates is selected with its demand recorded, the next highest $x$-coordinates value is recorded with its demand until the capacity constraints of the container is satisfied.

- The centroid of these points is found by taken the average of the extreme $x$-values and that of the $y$-values.

- The process is continued until all the initial centres are assigned.

\section{3) Updating of the cluster centers}

i) Compute distances from $C_{i}$ for all $X \in D$.

ii) Update the centre $C_{i}^{\prime}$ using Equation (16)

iii) If the difference between $\sum_{i=1}^{K}\left|C_{i}^{n-1}-C_{i}^{n-2}\right|$ and $\sum_{i=1}^{K}\left|C_{i}^{n}-C_{i}^{n-1}\right|$ is less than $\varepsilon$ stop else return to ii.

\section{4) Data Analysis \& Result Using the Proposed Model}

The data for the study was obtained jointly from Waste management division of Kumasi Metropolitan Assembly (KMA), Physical Planning unit of KMA, 
Ghana Statistical service (regional office) and a six (6) month continuous field work. The study area was divided into four zones due to physical boundaries such as streams, huge bridges which cannot be used by vehicles. Zone one 1) has seven hundred and forty-nine (749) houses with and has 1100 bins, zone two 2) with five hundred and sixty-one (561) houses has 828 bins, zone three 3) has five hundred and forty-two (542) houses with 792 bins and zone four had six hundred and twenty-three (623) houses with 789 bins of 140 liters respectively. The model was coded in $\mathrm{C}++$ and ran on Intel core $470 \mathrm{~Gb}$ computer.

\section{Cluster Centre from the Zones}

A $14 \mathrm{~m}^{3}$ skip containers were used for the location centers, with a compaction factor of 0.4 , a container can hold 166 of 140 litre bins. With the number of bins in zone one, the number of bins needed is calculated as $\frac{\sum_{i=1}^{n} q_{i}}{v_{j}}=\frac{1100}{166}=6.62 \cong 7$.

Coordinates of all the houses obtained by a Geographic Information System were taken and implemented using the probabilistic model. The final cluster (location centers) and their coordinates for each of the seven location centers by the model after 500 iterations is given in the tables below.

The maps mainly indicate the clusters obtained by the maximum insertion with their cluster centers where the waste collected should be sent to. The red color spots among the clusters of points represent the cluster centers for each cluster (which is differentiated by colors).

From Figure 2, there are seven sub-clusters in the seven bounded areas shown in the map below. Each sub-cluster serves users or customers in its bounded area. All the wastes collected in the area are sent to the associated sub-cluster center. The details of Figure 2 are stored in Table 1.

From Figure 3, there are five sub-clusters in for the five bounded areas shown in the map below. Each sub-cluster serves users or customers in its bounded area. All the wastes collected in the area is sent to the associated

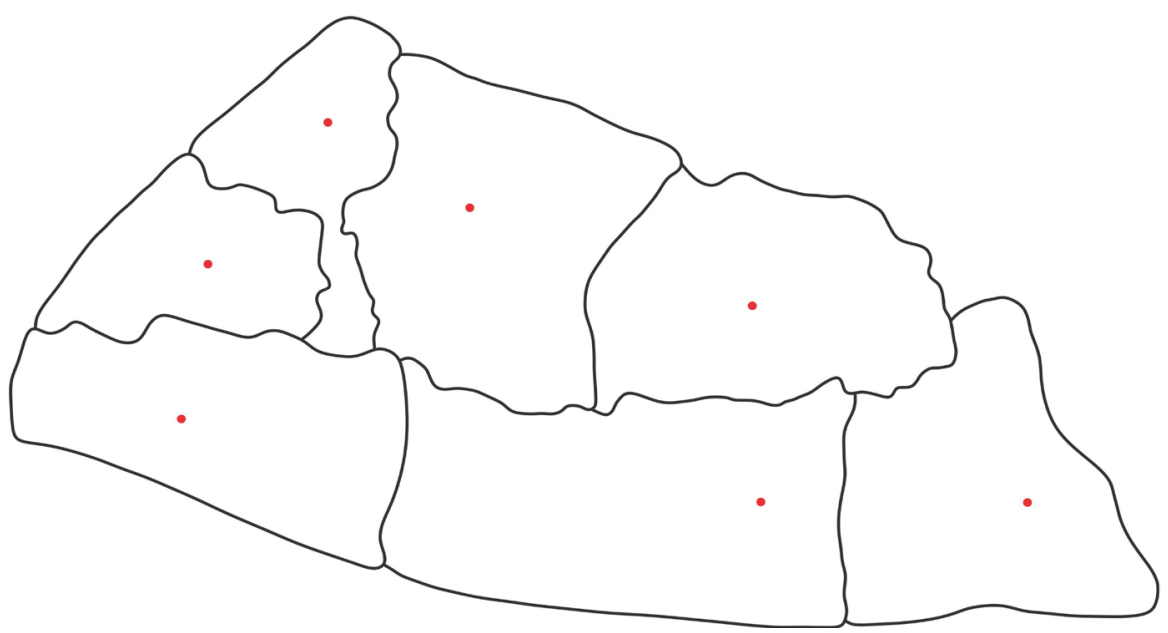

Figure 2. Final cluster centers in zone 1. 
Table 1. Summary of Zone 1 for Figure 2.

\begin{tabular}{ccccc}
\hline \multicolumn{5}{c}{ Zone 1 } \\
Sub-cluster & Cluster center & $\begin{array}{c}\text { Number of } \\
\text { customers }\end{array}$ & $\begin{array}{c}\text { Number of } \\
\text { bins (140I) }\end{array}$ & $\begin{array}{c}\text { Average distance (m) } \\
\text { from cluster center }\end{array}$ \\
\hline 1 & $(2836.52,3542.69)$ & 83 & 165 & 83.42 \\
2 & $(2806.72,3212.50)$ & 99 & 161 & 92.78 \\
$\mathbf{3}$ & $(2995.70,3822.21)$ & 85 & 165 & 79.88 \\
$\mathbf{4}$ & $(3188.21,3653.63)$ & 113 & 166 & 85.71 \\
$\mathbf{5}$ & $(3566.97,3458.67)$ & 136 & 166 & 90.89 \\
$\mathbf{6}$ & $(3578.23,3068.46)$ & 130 & 152 & 96.80 \\
7 & $(3936.45,3067.07)$ & 103 & 125 & 88.08 \\
& Total & $\mathbf{7 4 9}$ & 1100 & $\mathbf{8 8 . 2 2}$ \\
\hline
\end{tabular}

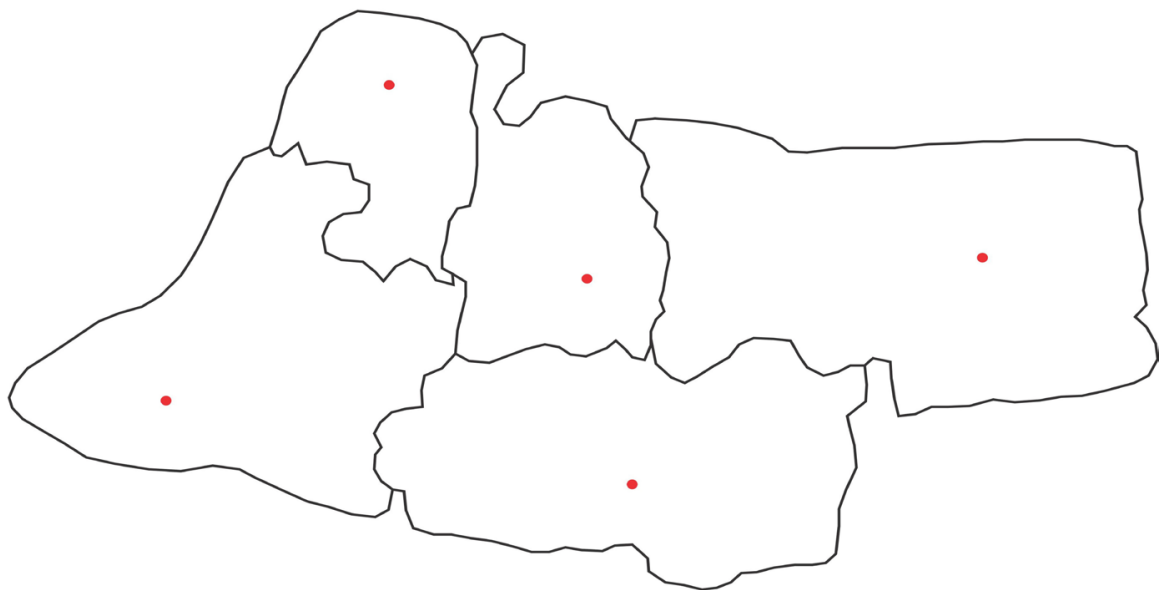

Figure 3. Final cluster centers in zone 2.

sub-cluster center. The detail of Figure 3 is stored in Table 2.

From Figure 4, there are five sub-clusters in for the five bounded areas shown in the map below. Each sub-cluster serves users or customers in its bounded area. All the wastes collected in the area are sent to the associated sub-cluster center. The detail of Figure 4 is stored in Table 3.

From Figure 5, there are five sub-clusters in for the five bounded areas shown in the map below. Each sub-cluster serves users or customers in its bounded area. All the wastes collected in the area are sent to the associated sub-cluster center. The detail of Figure 5 is stored in Table 4.

This table contains the summary of sub-cluster centers, number of customers in a sub-cluster and the number of bins used by customers in each sub-cluster in Zone 1. It is associated to Figure 2 and also contains the average distance from travelled from any location in the bounded area to its associated sub-cluster center. The cluster center points recorded in this table represents the geographical locations of each sub-cluster given by the maximum insertion method in and it is labeled with a red point in Figure 2. The cluster centers serve all 
Table 2. Summary of Zone 2 for Figure 3.

\begin{tabular}{lllll}
\hline \multicolumn{2}{c}{ Zone 2 } & & \\
\hline 2 & $(3636.30,3874.95)$ & 141 & 165 & 108.43 \\
3 & $(3962.67,4287.58)$ & 134 & 166 & 87.43 \\
4 & $(3589.01,4240.61)$ & 99 & 166 & 94.32 \\
$(3399.84,4593.46)$ & 84 & 166 & 79.28 \\
\hline
\end{tabular}

Figure 4. Final cluster centers in zone 3.

Table 3. Summary of Zone 3 for Figure 4.

\begin{tabular}{lrccc}
\hline \multicolumn{5}{c}{ Zone 3 } \\
\hline $\mathbf{1}$ & $(3533.30,4751.13)$ & 84 & 165 & 112.35 \\
$\mathbf{2}$ & $(4061.14,5251.59)$ & 134 & 139 & 98.56 \\
$\mathbf{3}$ & $(3680.54,5224.59)$ & 98 & 166 & 90.21 \\
$\mathbf{4}$ & $(3944.08,4603.17)$ & 124 & 164 & 97.18 \\
$\mathbf{5}$ & $(3690.92,4966.87)$ & 102 & 158 & 96.33 \\
& Total & $\mathbf{5 4 2}$ & $\mathbf{7 9 2}$ & $\mathbf{9 8 . 9 2}$ \\
\hline
\end{tabular}

users or customers in the bounded area. Thus, wastes collected in each bounded area in Zone 1 are sent to the sub-cluster center. There are seven sub-clusters in Zone 1 and labeled 1 - 7 in the table and in Figure 2.

This table contains the summary of sub-cluster centers, number of customers in a sub-cluster and the number of bins used by customers in each sub-cluster in Zone 2. It is associated to Figure 3 and also contains the average distance from travelled from any location in the bounded area to its associated sub-cluster center. The cluster center points recorded in this table represents the geographical locations of each sub-cluster given by the maximum insertion method in and it is labeled with a red point in Figure 3. The cluster centers serve all 


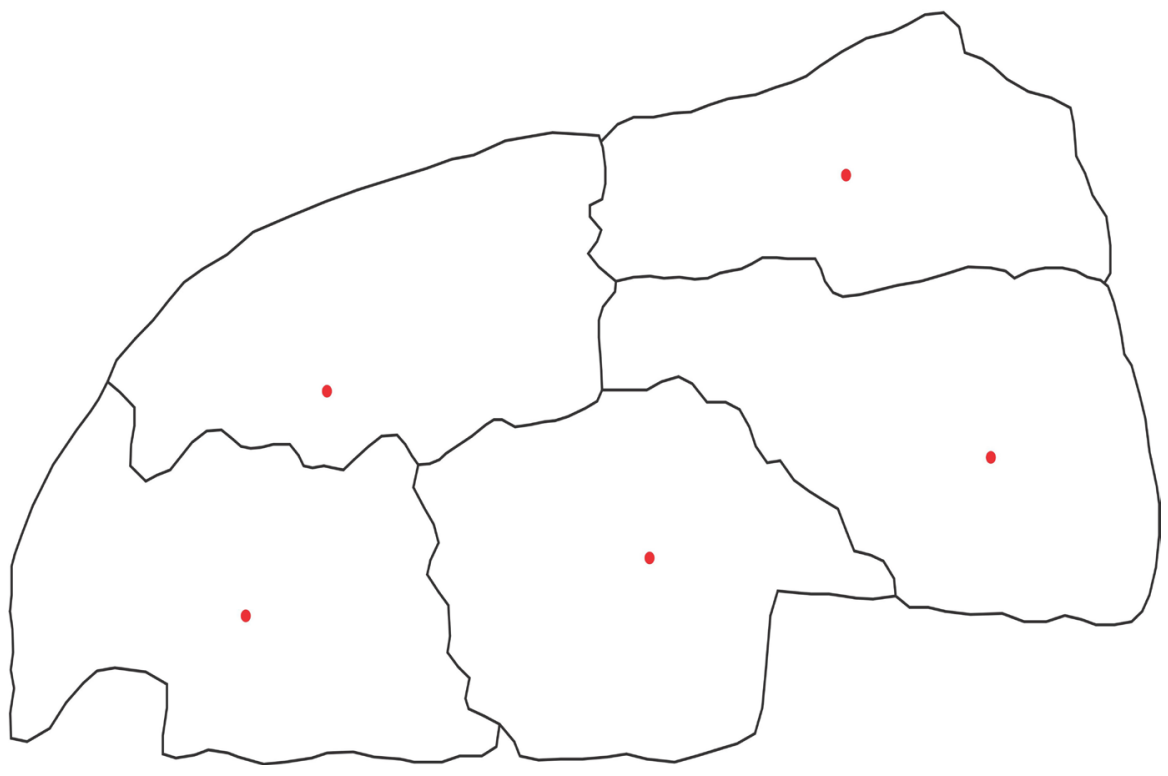

Figure 5. Final cluster centers in zone 4.

Table 4. Summary of Zone 4 for Figure 5.

\begin{tabular}{|c|c|c|c|c|}
\hline \multicolumn{5}{|c|}{ Zone 4} \\
\hline 1 & $(4313.19,6400.44)$ & 102 & 158 & 88.65 \\
\hline 2 & $(4470.51,5961.12)$ & 156 & 166 & 95.22 \\
\hline 3 & $(4116.35,5790.22)$ & 119 & 135 & 93.76 \\
\hline 4 & $(3792.16,6061.22)$ & 106 & 166 & 96.59 \\
\hline 5 & $(3705.55,5700.23)$ & 140 & 164 & 92.47 \\
\hline \multicolumn{2}{|c|}{ Total } & 623 & 789 & 93.34 \\
\hline \multicolumn{2}{|c|}{ Overall Total } & 2475 & 3509 & \\
\hline
\end{tabular}

users or customers in the bounded area. Thus, wastes collected in each bounded area in Zone 2 are sent to the sub-cluster center. There are five sub-clusters in Zone 2 and labeled 1 - 5 in the table and in Figure 3.

This table contains the summary of sub-cluster centers, number of customers in a sub-cluster and the number of bins used by customers in each sub-cluster in Zone 3. It is associated to Figure 4 and also contains the average distance from travelled from any location in the bounded area to its associated sub-cluster center. The cluster center points recorded in this table represents the geographical locations of each sub-cluster given by the maximum insertion method in and it is labeled with a red point in Figure 4 . The cluster centers serve all users or customers in the bounded area. Thus, wastes collected in each bounded area in Zone 3 are sent to the sub-cluster center. There are five sub-clusters in Zone 3 and labeled 1 - 5 in the table and in Figure 4.

This table contains the summary of sub-cluster centers, number of customers in a sub-cluster and the number of bins used by customers in each sub-cluster in Zone 4. It is associated to Figure 5 and also contains the average distance from 
travelled from any location in the bounded area to its associated sub-cluster center. The cluster center points recorded in this table represents the geographical locations of each sub-cluster given by the maximum insertion method in and it is labeled with a red point in Figure 5. The cluster centers serve all users or customers in the bounded area. Thus, wastes collected in each bounded area in Zone 4 are sent to the sub-cluster center. There are five sub-clusters in Zone 4 and labeled $1-5$ in the table and in Figure 5.

\section{Conclusion}

The adoption of the improved probabilistic distance location model to the study area of 2475 houses and about 3509 of 140 litre bins has shown that the area needs twenty-two (22) skip containers, seven in zone one (1), five skip containers each in zones two (2), three (3), and four (4) respectively as compared to the current 15 skip containers in the area. The model has also clearly identified the required number of customers to be assigned to each skip container based on the capacity of each customer and the capacity of the skip container. Our results have given an average walking distance of $92.86 \mathrm{~m}$ for a customer to access the skip container as compared to the existing average of $234.71 \mathrm{~m}$ to access a skip container.

\section{Conflicts of Interest}

The authors declare no conflicts of interest regarding the publication of this paper.

\section{References}

[1] Barbati, M. (2013) Models and Algorithms for Facility Location Problems with Equity Considerations.

[2] http://78.47.45.183/agency/ultimate-fm/20180215/72633487/filth-in-tafo-ahenbron om-as-residents-fear-outbreak-of-diseases

[3] Shiripour, S., Mahdavi, I., Amiri-Aref, M., Mohammadnia-Otaghsara, M. and Mahdavi-Amiri, N. (2012) Multi-Facility Location Problems in the Presence of a Probabilistic Line Barrier: A Mixed Integer Quadratic Programming Model. International Journal of Production Research, 50, 3988-4008.

https://doi.org/10.1080/00207543.2011.579639

[4] Erkut, E. and Neuman, S. (1989) Analytical Models for Locating Undesirable Facilities. European Journal of Operational Research, 40, 275-291.

https://doi.org/10.1016/0377-2217(89)90420-7

[5] Erkut, E. and Neuman, S. (1992) A Multiobjective Model for Locating Undesirable Facilities. Annals of Operations Research, 40, 209-227.

https://doi.org/10.1007/BF02060478

[6] Drezner and Wesolowsky, G.O. (1983) The Location of an Obnoxious Facility with Rectangular Distance. Journal of Regional Science, 23, 241-248.

https://doi.org/10.1111/j.1467-9787.1983.tb00800.x

[7] Wyman, M. and Kuby, M. (1993) A Multiobjective Location-Allocation Model for Assessing Toxic Waste Processing Technologies. Studies in Locational Analysis, 4, 
193-196

[8] Wyman, M. and Kuby, M. (1995) Proactive Optimization of Toxic Waste Transportation, Location and Technology. Location Science, 3, 167-185. https://doi.org/10.1016/0966-8349(95)00014-3

[9] Ghiani, G., Laganà, D., Manni, E. and Triki, C. (2012) Capacitated Location of Collection Sites in an Urban Waste Management System. Waste Management, 32, 1291-1296. https://doi.org/10.1016/j.wasman.2012.02.009

[10] Costa, M.G., Captivo, M.E. and Clímaco, J. (2008) Capacitated Single Allocation Hub Location Problem-A Bicriteria Approach. Computers \& Operations Research, 35, 3671-3695. https://doi.org/10.1016/j.cor.2007.04.005

[11] Cappanera, P., Gallo, G. and Maffioli, F. (2004) Discrete Facility Location and Routing of Obnoxious Facilities. Discrete Applied Mathematics, 133, 3-28. https://doi.org/10.1016/S0166-218X(03)00431-1

[12] Fonseca, M.C. and Captivo, M.E. (2007) Analysis of Bicriteria Semi Obnoxious Location Models. Paper Presented at Tercer Encuentro de la Red Iberoamericana de Evaluación Decisión Multicriterio, R.E.D.-M. 2007, Culiacán.

[13] Shen, Z., Zhan, R. and Zhang, J. (2011) The Reliable Facility Location Problem: Formulations, Heuristics, and Approximation Algorithms. INFORMS Journal on Computing, 23, 470-482. https://doi.org/10.1287/ijoc.1100.0414

[14] Li, X., Tomasgard, A. and Barton, P.I. (2011) Nonconvex Generalized Benders Decomposition for Stochastic Separable Mixed-Integer Nonlinear Programs. Journal of Optimization Theory and Applications, 151, 425. https://doi.org/10.1007/s10957-011-9888-1

[15] Li, Q., Zeng, B. and Savachkin, A. (2012) Reliable Facility Location Design under Disruptions. Computers \& Operations Research, 40, 901-909. https://doi.org/10.1016/j.cor.2012.11.012

[16] An, Y., Zeng, B., Zhang, Y. and Zhao, L. (2014) Reliable p-Median Facility Location Problem: Two-Stage Robust Models and Algorithms. Transportation Research Part B: Methodological, 64, 54-72. https://doi.org/10.1016/j.trb.2014.02.005

[17] An, Y., Zhang, Y. and Zeng, B. (2015) The Reliable Hub-and-Spoke Design Problem: Models and Algorithms. Transportation Research Part B: Methodological, 77, 103-122. https://doi.org/10.1016/j.trb.2015.02.006

[18] Farahani, R.Z., SteadieSeifi, M. and Asgari, N. (2010) Multiple Criteria Facility Location Problems: A Survey. Applied Mathematical Modelling, 34, 1689-1709. https://doi.org/10.1016/j.apm.2009.10.005 